\title{
Editorial
}

\section{Getting an air lock on the macular hole}

Anyone who has tried amateur plumbing, especially in connection with central heating, will be familiar with the power of the air lock. It is really remarkable how air is able to block the flow of water in a pipe, the lumen of which is enormous compared with the minute orifice through which air itself can pass, for example in a slow puncture of a motor tyre. It is all supposed to be something to do with surface tension, but I still regard it as one of the wonders of nature, and it puts me off trying to do the plumbing except in the most dire emergencies. Since the power of air in blocking pipes has been known for so long, and since a hole is after all only a very short pipe, it is remarkable that it took so long for the potential of air (or other gases) in the treatment of retinal detachment to be realised.

One of the minor mysteries of retinal detachment has always been the macular hole. In the days before binocular indirect ophthalmoscopy and the various slit-lamp techniques for retinal examination it was difficult to establish whether a macular hole was genuine. If the macula was flat there was less of a problem, but if it was detached the illusion of a macular hole was nearly always present when a direct ophthalmoscope was used. This led to the general belief that macular 'holes' could be ignored and the search concentrated on the rest of the retina.

With the better methods of examination now available it has become clear that true macular holes do exist and can cause retinal detachment. Nevertheless a detachment due to an isolated macular hole is still very much of a rarity. This is fortunate for several reasons, not least because some degree of central visual loss is almost inevitable even after successful retinal replacement. Destruction of the macula by photocoagulation, or by cryo or even diathermy, if the macula can be reached, has normally been attempted as part of the operation for replacement, and a number of ingenious devices for achieving an indent or buckle have been suggested over the years. It was always a struggle to place the chosen method of indentation far enough back on the eye, whether it was Rosengren's ball or solid or sponge silicone rubber, and it was particularly awkward to insert the most posterior stitches. I believe Kronlein's lateral orbitotomy was even suggested at some stage as a means of reaching far enough posteriorly on the globe.

A paper by Yoon-Kee Lai in this issue reminds us of the revolution which seems to have occurred in the treatment of detachment due to macular hole. The struggles and difficul- ties outlined above are now apparently things of the past. The paper does not claim that the technique is original but it is nevertheless valuable for it to be brought to our attention, since many non-specialised ophthalmologists still carry out a number of detachment procedures, and this technique should prove extremely useful in these rare cases.

It is interesting to speculate on why it has taken so long for the idea of blocking holes in the retina with intravitreal gas to catch on with ophthalmologists. There must be a number of reasons, not least of which would be the traditional reluctance of ophthalmologists in the first half of the twentieth century to have anything to do with the vitreous if they could avoid it. The vitreous was almost taboo. Other reasons were the difficulties of working out a good sequence for applying heat, light, or cryo, draining fluid, raising a buckle, and inserting gas without completely losing the ability to see what was going on inside the eye. There is some interesting correspondence on the subject in the $B F O$ of November $1986,{ }^{12}$ and one of the authors concerned pointed out that in the D-ACE sequence (drainage, air, cryo, explant) the air tamponade allowed a more modest buckle to be employed than might otherwise have been needed. Macular hole tamponade carries this principle further, since in the cases described in the present paper neither buckle nor choroidoretinal burning was used.

To revert to my thoughts on plumbing expressed at the beginning of this editorial, it is indeed fortunate that air does not pass through the macular hole but, by blocking it in the same way as it blocks my central heating system when I try to tamper with it, completely cuts off the subretinal fluid from the vitreous, thus allowing rapid resorption. The remarkable thing is that no burning is needed to get the retina to adhere, though there is of course a precedent for this as was pointed out by Chignell and Markham ${ }^{3}$ when they did a series of conventional buckling procedures without cryo, with fairly good results. Such daring surgery would not normally be advisable now for the more usual type of detachment with peripheral tears, but it is certainly well worth knowing that it is possible to get away with it in cases with a macular hole.

REDMOND SMITH

1 Urrets-Zavalia A. Treatment of retinal detachment. Br $\mathcal{F}$ Ophthalmol 1986; 70: 874.

2 McLeod D. Reply to Urrets-Zavalia. Brf Ophthalmol 1986; 70: 875.

Chignell A, Markham RHC. Retinal detachment surgery without cryotherapy. BrF Ophthalmol 1981; 65: 371-3. 